\title{
The fibrillar organisation of the osteon and cellular aspects of its development
}

\author{
A morphological study using the SEM fractured cortex technique
}

\author{
Ugo E. Pazzaglia • Terenzio Congiu • \\ Guido Zarattini · Marcella Marchese • \\ Daniela Quacci
}

Received: 15 September 2010/ Accepted: 14 November 2010/Published online: 7 January 2011

(C) Japanese Association of Anatomists 2010

\begin{abstract}
The collagen architecture of secondary osteons was studied with scanning electron microscopy (SEM) employing the fractured cortex technique and osmic maceration. Fibrillar orientation and the change in their direction in sequential lamellae was documented where lamellar formation was ongoing, as well as in resorption pits where osteoclasts had exposed the collagen organisation of the underlying layers. Applying an adaptive stereo matching technique, the mean thickness of matrix layers removed by osteoclasts was $1.36 \pm 0.45 \mu \mathrm{m}$. It was also documented that osteoclasts do not attack the cellular membrane of the exposed osteocytes. The mean linear osteoblast density in fractured hemicanals was assessed with SEM and no significant differences were observed comparing larger with smaller central canal osteons. These findings suggested a balance between the differentiated osteoblasts that have aligned on the surface of the cutting cone and those that are transformed into osteocytes, because the canal surface is progressively reduced as the lamellar apposition advances.
\end{abstract}

Keywords Osteon - Fibril organization .

Bone remodelling · Osteocyte $\cdot$ Osteoblast $\cdot$ Osteoclast

U. E. Pazzaglia $(\bowtie) \cdot$ G. Zarattini · M. Marchese

Clinica Ortopedica dell'Università di Brescia,

Spedali Civili di Brescia, 25123 Brescia, Italy

e-mail: ortopedia2@spedalicivili.brescia.it

T. Congiu - D. Quacci

Dipartimento di Morfologia Umana,

Università dell'Insubria, 21100 Varese, Italy

e-mail: terenzio.congiu@uninsubria.it

\section{Introduction}

Since the first observations of Gebhardt (1906) and Weidenreich (1923), the fibrillar pattern of human secondary osteons has been the subject of extensive investigation. This has been aided by the evolution of new techniques (Ascenzi et al. 1983; Ascenzi and Bonucci 1965, 1968; Giraud-Guille 1988; Smith 1963; Wagermaier et al. 2006; Weiner et al. 1999). Two models have been proposed, the first confirming the early interpretation of Gebhardt, with the collagen fibrils orientated in parallel in each lamella with a different direction in each neighbouring lamella (Ruth 1953; Smith 1960; Giraud-Guille 1988); the second interpreting the different aspects of alternate lamellae with the density of collagen fibrils and of mineral elements rather than with their orientation (Boyde and Hordell 1969; Frank et al. 1955; Rouiller et al. 1952; Ruth 1953; Ziegler 1906). Marotti (1993), examining with SEM prismatic samples of human cortex in both cross and longitudinal section, observed the same configuration whatever the plane of the section. As such, he consequently interpreted bone lamellation as a different density of the fibrillar matrix without an interlamellar cementing substance.

The fibrillar organisation inside the osteon has been related to the spatial disposition of osteoblasts and to the transformation of the latter into osteocytes (Marotti 1973; Marotti et al. 1992; Palumbo 1986; Palumbo et al. 1990); however, the change in fibril orientation in the lamellar sequence cannot be explained on the basis of the activity of single osteoblasts. On the contrary, this structural pattern is consistent with the synchronous and phasic activity of a pool of osteoblasts (Pazzaglia et al. 2010).

To study the relationship between the fibrillar organisation of the osteon and the cells involved in its development, we used the SEM fractured cortex technique 
(Pazzaglia et al. 2009a, b). We examined the architecture of the fibrillar system both in the phase of its formation from the inside of the vascular canal as well as during the process of remodelling, when the osteoclasts remove layers of already structured matrix in front of the advancing cutting cones. With this approach it was also possible to show the spatial relationship of the cells with the surrounding matrix in either the active phase of apposition (osteoblasts), the steady state (osteocytes) or the resorption process (osteoclasts).

The application of the SEM fractured surface technique to the study of cortical bone structure has provided new insights into the orientation of collagen fibrils in sequential lamellae. It has also allowed the stratified mode of osteoclastic resorption and of the particular morphology of the osteocytes exposed by the resorbing cells to be examined. These findings have validated the twisted plywood model of lamellar bone and give new insights into bone cell function and activity.

\section{Materials and methods}

The study was carried out on the femurs of six male, New Zealand white rabbits (Charles River, Calco, Bergamo, Italy) with a body weight between 3.0 and $3.5 \mathrm{~kg}$. The rabbits were approximately 8 months of age. The care and use of experimental animals was consistent with the procedures and regulations set by the Italian Health Ministry.

The rabbits were anaesthetised with ketamine cloridrate (Imagel) and xylazine (Rompum). The aorta and the vena cava were exposed through a mid-line abdominal incision. A $1.5 \mathrm{~mm}$ catheter was inserted into the aorta in a proximal to distal direction between the diaphragm and the origin of the renal arteries. The aorta was then ligated tightly with two knots around the catheter and the rabbit was euthanised with a further dose of the anaesthetic just prior to initiating perfusion of the vascular tree. The rabbits were injected with $300 \mathrm{ml}$ of formaldehyde solution (2\%) with a hand syringe at a pressure of 150-200 mm mercury until the lower limbs were completely perfused. This process took approximately $5 \mathrm{~s}$. Due to the high pressure within the extracortical vascular tree, it was necessary to clamp the vena cava with artery forceps to even up the difference in resistance between the extracortical and intracortical systems prior to perfusion. Using this technique, complete perfusion of the intracortical sector vessel is achieved without dilation of the injected vessels (Pazzaglia et al. 1997).

The skin of the limbs was removed and both femurs were dissected from the soft tissues and stored in formaldehyde solution (2\%). The central part of the right femoral diaphysis (about $15 \mathrm{~mm}$ long) was cut with a hand saw in a plane perpendicular to the major axis of the bone and the proximal and distal parts discarded. The central cylinder of each right femur was split in a longitudinal direction by creating three or four fractures initiated by a notch made with a chisel on the transversally cut surface. The specimens were submitted to osmic maceration: they were washed in phosphate buffered saline (PBS, $\mathrm{pH} 7.2$ ) and then post-fixed in a solution of $1 \%$ osmium tetroxide and $1.25 \%$ potassium ferrocyanide for $2 \mathrm{~h}$. They were then washed in PBS, immersed in $0.1 \%$ osmium tetroxide in PBS for $12 \mathrm{~h}$ at room temperature and again washed in PBS. All the specimens were dehydrated in ascending grades of ethanol, subjected to critical point drying in $\mathrm{CO}_{2}$, coated with $10 \mathrm{~nm}$ gold palladium in a vacuum sputter Emitech K550 (Edax, Mahwah, NJ) and studied in the direct mode with a Philips XL 30 SEM-FEG scanning electron microscope (Philips, Eindhoven, the Netherlands). After observation, the same specimens were immersed in a bath of hydrogen peroxide (40\%) for $24 \mathrm{~h}$ to remove all cells and organic debris from the surface and again prepared for SEM observation.

The diaphyses of the left femurs were reduced to segments of about $3 \mathrm{~mm}$, embedded in methylmethacrylate and sections were cut in a plane perpendicular to the major axis of the bone with a motorised microtome. Slides were stained with solochrome, others were decalcified in EDTA solution $(10 \%)$ for 6 weeks at room temperature, embedded in paraffin, and transversal sections were stained with haematoxylin-eosine. The slides were observed with a light microscope Olympus BX 51 in bright field, in phase contrast and in polarised light.

\section{Morphometry}

In hemicanals exposed by fracture of the cortex and submitted to osmic maceration, the density of osteoblasts on the inner surface of secondary osteons that were completing their lamellar structure were evaluated using SEM. Of a total of 316 canals exposed in the six right femurs, 281 $(88.9 \%)$ did not present active osteoblasts or had been damaged by the mechanical stress of the fracture, therefore only 35 were suitable for measurement and cell counting. With the electron beam perpendicular to the surface of the hemicanal under examination, after tracing the mid longitudinal axis, perpendicular diameters at a regular distance of $50 \mu \mathrm{m}$ were assigned and measured (Black et al. 1974). It was arbitrarily assumed that the canal had a rounded shape and that the fracture had divided the canal into two symmetrical hemicanals: therefore correcting each diameter by a factor, $\pi$, this was considered as the length of the underlying hemicircumference. The number of osteoblasts intercepting each hemicircumference was counted and the linear density of cells calculated (Fig. 1). To compare the density of linear osteoblasts in canals of different developmental stages they were divided into two groups: the first 


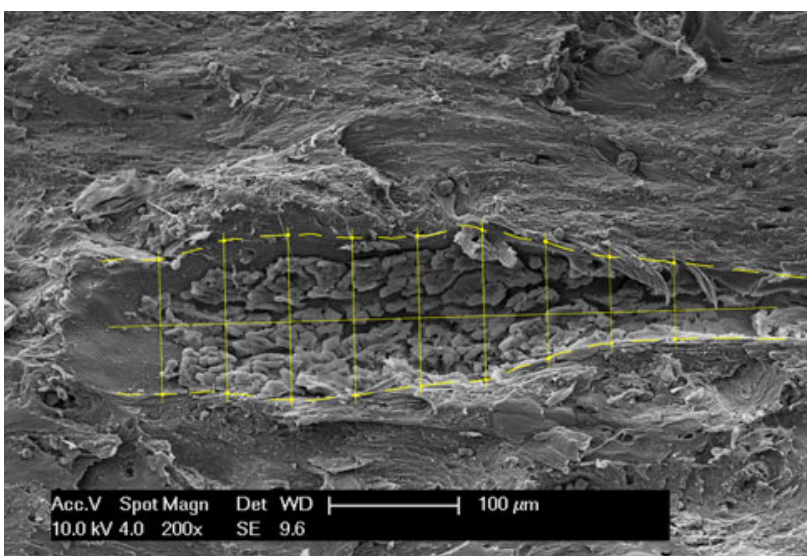

Fig. 1 Scanning electron microscopy (SEM) of a fractured surface of a hemicanal with a sheet of osteoblast lining the inner surface. Osteoblast linear density was assessed on hemidiameters perpendicular to the canal axis by counting the cells intercepted by each hemidiameter. Bar $100 \mu \mathrm{m}$

including all densities of hemicircumferences below $100 \mu \mathrm{m}$, corresponding to osteons in an advanced phase of lamellar apposition; the second of those over $200 \mu \mathrm{m}$, corresponding to an earlier phase of organisation. The limits of the two groups were set arbitrarily based on a study on Havers' canals morphometry carried out with SEM paired fractured surfaces (Pazzaglia et al. 2009a) because the fracture lines tend to run through the middle of the canal and never tangentially. Hemicircumferences between 100 and $200 \mu \mathrm{m}$ were excluded.

The thickness of the matrix layers exposed by the osteoclasts inside the cutting cones resorption pits was measured on images enlarged $80 \times$ with the electron beam perpendicular to the surface at a working distance of $10 \mathrm{~mm}$. The specimens were tilted clockwise and anticlockwise $10^{\circ}$ in the plane perpendicular to the border of the exposed lamella. The corresponding images of the borders $\left(+10^{\circ}\right.$ and $\left.-10^{\circ}\right)$ were acquired and processed with a stereometric program (Manelli et al. 2007).

\section{Statistical analysis}

The linear density of osteoblasts and the thickness of resorbed matrix layers were calculated as the mean of all the sections examined in each bone. Standard deviation referred to the group of six rabbits $(n=6)$. Linear osteoblast density of osteons with small and large vascular canal was compared with Student's $t$ test.

\section{Results}

The longitudinally fractured cortex of the right femurs exposed osteocytic lacunae, canalicula and sufficiently

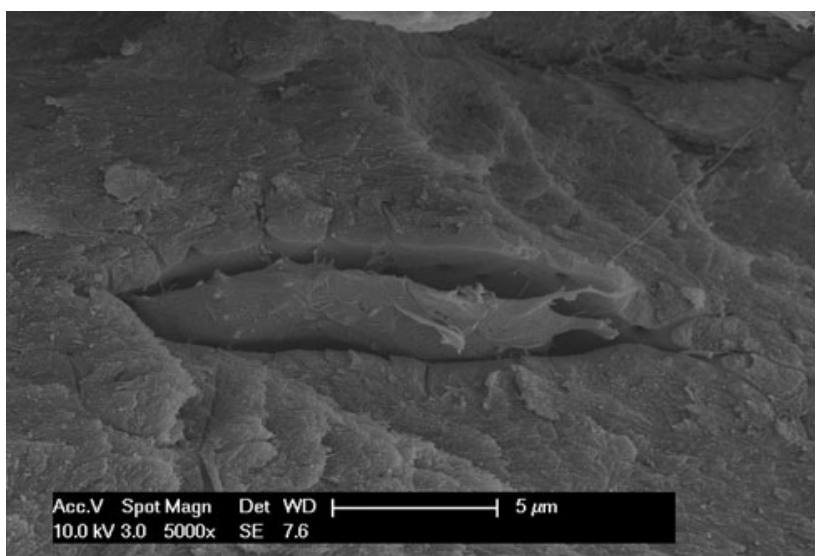

Fig. 2 SEM of the fractured surface of a hemilacuna inside its own osteocyte. The emerging cones of processes are present on both the flat surfaces and the perimeter of the cell. Some canalicula have been exposed by the fracture plane on both surfaces of the osteocytic lacuna. The membrane processes have been detached from the canalicula by shrinkage and mechanical stress. The canalicula have a large base and then become cylindrical. A bifurcation of a process and the corresponding canaliculum is evident on the right side. Bar $5 \mu \mathrm{m}$

long tracts of the vascular canals and of the cutting cones to perform a quantitative assessment of the osteoblasts lining the inner surface of the hemicanals. Mechanical damage due to the fracture strain force detached cells and other soft tissue structures from the exposed bone surface, therefore only $11.1 \%$ of the exposed hemicanals were suitable for osteoblasts linear density assessment. About the same percentage of osteocytic lacunae cells remained adherent to the wall and could be studied with SEM in direct mode.

The osteocytes in their own lacuna appeared flattened and lentiform in shape, and possessed processes arising from both surfaces and from the perimeter. These processes penetrated inside canalicula pertaining to the corresponding lacuna and progressively became thinner as they advanced in depth (Fig. 2). Osteoblasts appeared as polygonal flattened cells (Fig. 1) packed closely on the surface of canals where apposition was very active, and otherwise present in a more scattered disposition. The undersurface of the cell facing the bone was not so apparent, while the dome on the opposed side (vascular) was smooth and devoid of any processes. When the sheet of osteoblasts was removed by oxygen peroxide treatment or osmic maceration, the texture of collagen fibrils showed a clear spatial orientation and a change in direction of sequential lamellae was also evident (Fig. 3a, b).

The cutting cones exposed by the fracture showed that the osteoclasts did not deepen straight into the matrix, but rather advanced by removing superficial layers of collagenic matrix in limited sectors (Fig. 4a); the zonal aspect of the pits was suggestive of a phasic and discontinuous resorption. The sharp and multi-layered border of the pits 


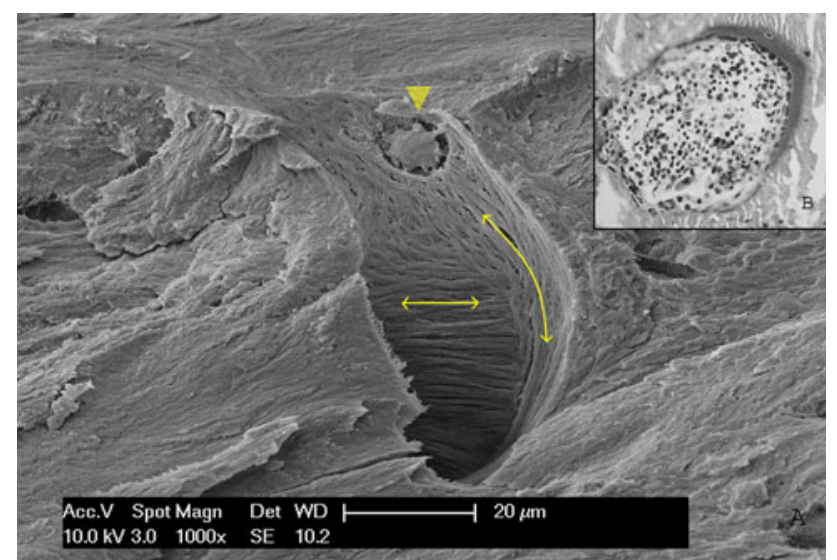

Fig. 3 a SEM of a fractured surface of hemicanal (connecting the marrow space with the haversian system), showing the overlapping of sequential lamellae with different fibrillar polarisation (long arrows). An osteoblast is shown in the process of becoming incorporated into the bone matrix (arrowhead). b Light microscopy in bright field, undecalcified solochrome-stained section. The incomplete osteoblast row in transverse section can explain the overlapping of fibril layers with different orientation as observed in the SEM picture. Bars a $20 \mu \mathrm{m}, \mathbf{b} 100 \mu \mathrm{m}$

provided further evidence of the variation in collagen fibril orientation in the overlying lamellae (Fig. 4b). Due to the phasic resorption, it was also possible to observe sequences of exposed layers of matrix (Fig. 4c) and, by applying the stereometric technique to the sharp borders, to measure the thickness, which was $1.36 \pm 0.45 \mu \mathrm{m}$.

The osteoclasts appeared to resorb the mineralised matrix layer by layer, but when, in the course of this process, they open an osteocytic lacuna, no damage of the osteocyte membrane is evident (Fig. 5). The resorption pits and cells of the osteogenic line showed a definite location, distribution and density in the cutting cones sectioned by the fracture (Fig. 6):

- Zone A: a cutting head with typical resorption pits, recently exposed osteocytes and old canals intercepted by the advancing cutting cone;

- Zone B: several osteocytes/osteoblasts scattered on the surface, but not densely packed. There has been apposition of a thin layer of new matrix, because the chopped collagen fibrils (typical of pits) were no longer evident;

- Zone C: the whole canal is surface covered by a sheet of densely packed osteoblasts with the typical features of the appositional front.

The mean linear osteoblast density was compared between two classes of canal sizes: the first (osteon central canal diameters $<100 \mu \mathrm{m})$ and the other $(>200 \mu \mathrm{m})$. These classes were selected as representative of osteons that had nearly completed their lamellar structure or of those in an
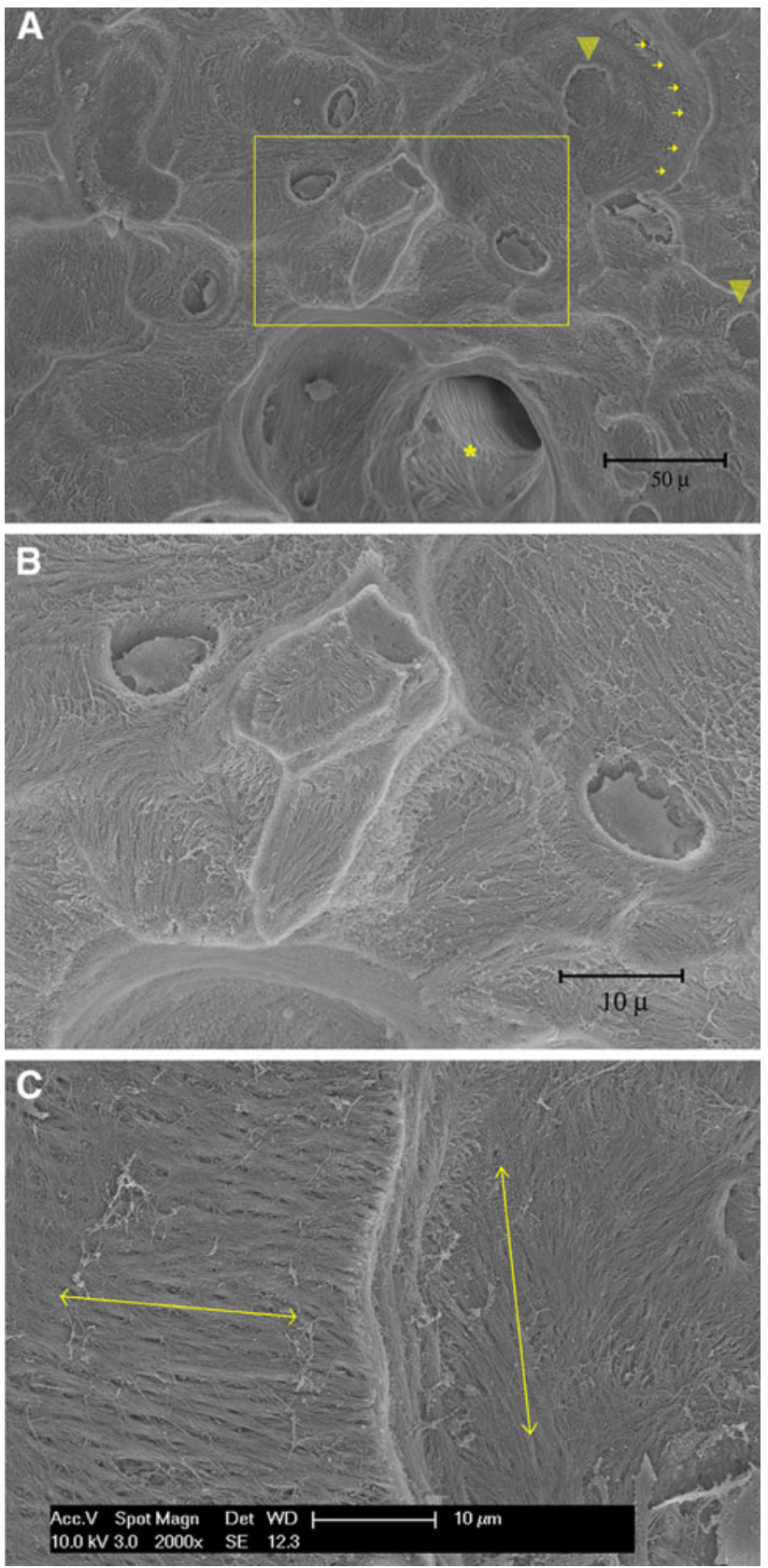

Fig. 4a-c SEM of a fractured surface of a wide resorption pit. Excavations were carried out at different depth revealing the stratification of the lamellar structure and the different direction of the collagen fibrils in sequential lamellae (arrows). Several osteocytes have been exposed, but their cellular membrane is intact. No processes are present on the exposed surface of osteocytes. Remnants of empty lacunae are also present (arrowheads) and a canal intercepted by the advancing cutting cone (asterisk). b Enlarged detail of the box in a showing the sharp border of the resorption pits, the lamellar stratification and a partially resorbed osteocytic lacuna (arrow). c Lamellar stratification and polarisation of the fibrillar pattern (long arrows). Bars a $50 \mu \mathrm{m} ; \mathbf{b}, \mathbf{c} 10 \mu \mathrm{m}$

earlier phase of the osteonal development, when the central canal is still large. The mean linear osteoblast density was $0.069 \pm 0.0059$ and $0.066 \pm 0.0058 ; P=0.028$. 


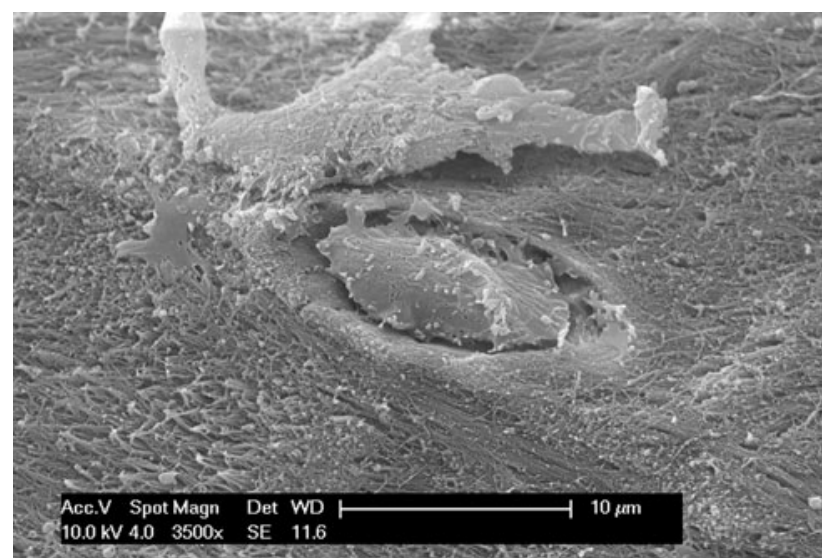

Fig. 5 An osteocyte within its own lacuna has been exposed by osteoclasts in a resorption pit, as evidenced by the tip of the cut collagen fibrils in the lower half of the figure. In the top half renewed apposition by recruited osteoblasts is beginning

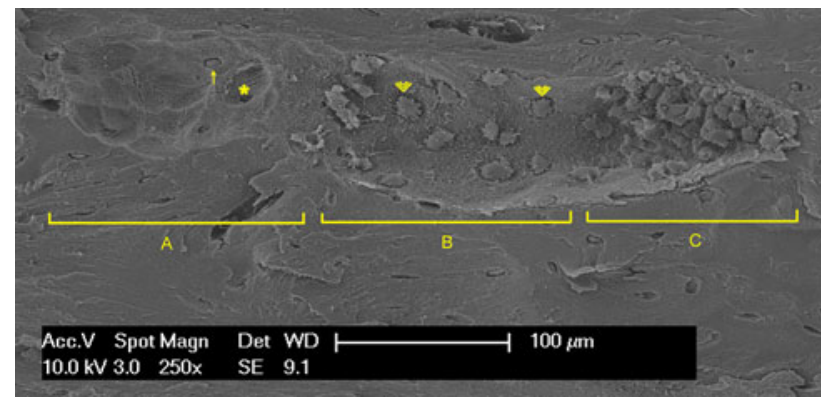

Fig. 6 SEM of a fractured surface of a hemi-cutting cone. Zones: A Cutting head with pits formed by osteoclasts-a recently exposed osteocyte is evident (small arrow) and a vascular canal has been intercepted by the advancing cone (asterisk); $B$ scattered osteoblasts have started to lay down the first layer of matrix, because the floor of the canal is smooth (cf. structure of pits in zone A) - some osteoblasts have started to sink within their own lacuna (arrowheads); $C$ higher density of osteoblasts typical of structuring osteon. Bar $100 \mu \mathrm{m}$

\section{Discussion}

Scanning electron microscopy of the fractured surfaces of cortical bone documented osteoblast apposition on the inner surface of the osteon's central canal, and osteoclasts resorption of the cutting cones. To the best of our knowledge this technical application is original and has enabled the fibrillar organisation and the cell-matrix relationship in compact bone remodelling to be studied. The organisation of lamellar collagen fibrils has been the subject of debate for many years (Gebhardt 1906; Rouiller et al. 1952; Ruth 1953; Ziegler 1906). The currently favoured model is that concentrical layers of parallel fibrils vary their orientation in sequential planes (Frasca et al. 1977; Raspanti et al. 1994, 1996; Reid 1986). Our observations would support this hypothesis. However, the deductions of Marotti and Muglia (1988) and Marotti (1993) support a model of alternating collagen-rich (dense lamellae) and collagenpoor (loose lamellae) layers, all having an interwoven arrangement of fibres.

Artefacts are certainly produced whatever microscopic technique is applied. The technique of creating fractures in the cortical bone used in this study produced a severe strain on the calcified bone matrix, on the cells of the matrix, and on the vascular structures contained within it. As such, the percentage of bone surface that remained useful for observation was consequently reduced. Despite this, qualitative artefacts due to fixation and coating remained the same as for any other application of SEM. In any case, the mechanical artefacts produced were less than would be expected if the specimens were prepared using the sawing and grinding techniques currently employed in the preparation of bone for SEM examination.

The ordered orientation of fibrils in sequential lamellae has been documented at sites where lamella formation was ongoing, and it has been correlated with the locomotor strain direction in cortical bone (Riggs et al. 1993a, b). The lamellar thickness, or better, the half interlamellar separation, has been measured by several authors (Frost 1962; Ascenzi et al. 1982; Kragstrub et al. 1983; Reid 1986) with values ranging from 1.94 to $3.63 \mu \mathrm{m}$ in the femoral cortex of normal young subjects (Ascenzi et al. 1982). At sites of resorption, we observed that osteoclasts acted as scrapers, removing layers of collagenic matrix in sequence. The mean thickness of these layers was less than in any type of measured lamella. This was not unexpected because it represents the depth of the osteoclastic action on the bone surface, whereas lamellae are the product of the synchronous deposition of a sheet of osteoblasts lining the inner cavity of the forming osteon. The stratification evident in the resorption pits, however, also supported a model of a flat and regular parallelism of the fibrils of each lamella, with a change of direction in sequential lamella.

The fibrils appeared to have been assembled and oriented in compliance with a phasic rule, because the apposition of a new layer of collagen fibrils with different polarity requires the synchronous change of orientation of all the fibrils present in that layer. Variations in lamellar thickness can be explained by the different lengths of each phase, but what remains unexplained at present is the synchronism of the change of direction and the factors controlling this type of organisation. However, a resting phase of the pool of osteoblasts could serve to remodulate their network of processes before starting to form a new lamella (Pazzaglia et al. 2010).

The resorption pits in front of the advancing cutting cone have been only scarcely studied with SEM as yet because of the difficulty in exposing these cavities. The fractured cortex technique allowed a large surface area of the cutting cone to be observed, thus enabling us to 
document that the osteoclasts do not advance straight ahead within the depths of the compact bone, but rather they resorb the underlying matrix layer by layer. This is no different from what has been observed to occur on the surface of bone trabeculae or with the same type of cells cultured in vitro on bone slices (Chambers et al. 1984; Fuller et al. 1994). Osteoclasts are known to concentrate $\mathrm{H}^{+}$ions at the level of the brush border membrane, which serves to remove hydroxyapatite crystals from the calcified matrix and to produce proteolytic enzymes, which then digest the collagen fibrils of the matrix (Bonucci 2007). However, little is known about the selectivity of osteoclastic resorption. A transmission electron microscopy study carried out in developing calvaria suggested that osteoclasts engulf and destroy osteocytes during bone resorption (Elmardi et al. 1990); our SEM findings on the contrary documented the resorption of the peri-lacunar matrix but without damage to the cellular membrane of the exposed osteocytes. In the cortical bone cutting cones, active osteoblasts are observed to initiate the concentric lamellar apposition behind the osteoclasts of the cutting head. The origin of the osteogenic cells, however, has never been questioned, because it has always been taken for granted that the vascular loop or the central vessel were the unique sources. The observation of healthy osteocytes in the resorption pits of the cutting head suggests the possibility that a contribution to the pool of osteoblasts could come from osteocytes exposed by osteoclastic resorption. If the latter hypothesis is confirmed by further studies, it would have the general implication that the inverse transformation from osteocyte to osteoblast occurs regularly in secondary remodelling of cortical bone.

The osteoblasts in sector B represent the first layer behind the cutting head and their position is labelled by the initial formation of the new lacuna, therefore it can be excluded that the observed cells distribution would be an artefact secondary to mechanical dragging. The number of osteoblasts on the surface increased from sector B to sector C. Densitometrical parameters of osteogenic surfaces have been used to evaluate the osteoblast-osteocyte relationship (Marotti et al. 1992); however, these measurements have been carried out on the growing surface of the long bones marrow canal, which, for several reasons, represents a different environment from the central canal of the osteon (Pazzaglia et al. 2009a, b). The SEM fractured cortex technique enabled direct access to the intracortical canals and made this type of morphometric analysis possible. As the internal surface of the cutting cone is progressively reduced with the advancement of the lamellar apposition, the finding that there is no significant difference in linear osteoblast density between canals of large and small diameter may suggest a balance between the differentiated osteoblasts that have aligned on the canal surface and those transformed into osteocytes, thus maintaining a constant surface density of osteoblasts throughout the osteon closure process.

Acknowledgements The study was carried out using a scanning electron microscope from the "Centre Great Instruments" of Insubria University, and was supported by research funds of Brescia University. The authors acknowledge Professor M. Raspanti for his suggestions and advice in applying the stereometric method.

\section{References}

Ascenzi A, Bonucci E (1965) An electron microscope study of osteon calcification. J Ultrastruct Res 12:287-303

Ascenzi A, Bonucci E (1968) The compressive properties of single osteons. Anat Rec 161(3):377-391

Ascenzi A, Benvenuti A, Bonucci E (1982) The tensile properties of single osteonic lamellae: technical problems and preliminary results. J Biomech 15:29-37

Ascenzi A, Bigi A, Ripamonti A, Roveri N (1983) X-ray diffraction analysis of transversal osteonic lamellae. Calcif Tissue Int 35(3):279-283

Bonucci E (2007) Biological calcification: normal and pathological processes in the early stages. Springer, Heidelberg

Black J, Mattson R, Korostoff E (1974) Haversian osteons: size, distribution, internal structure, and orientation. J Biomed Mater Res 8(5):299-319

Boyde A, Hordell MH (1969) Scanning electron microscopy of lamellar bone. Z Zellforsch Mikrosk Anat 93(2):213-231

Chambers TJ, Revell PA, Fuller K, Athanasou NA (1984) Resorption of bone by isolated rabbit osteoclasts. J Cell Sci 66:383-399

Elmardi AS, Katchburian MV, Katchburian E (1990) Electron microscopy of developing calvaria reveals images that suggest that osteoclasts engulf and destroy osteocytes during bone resorption. Calcif Tissue Int 46:239-245

Frank R, Frank P, Klein M, Fontaine R (1955) Electronic microscopy of normal human compact bone. Arch Anat Pathol (Paris) 44(2):191-206

Frasca P, Harper RA, Katz JL (1977) Collagen fiber orientations in human secondary osteons. Acta Anat (Basel) 98(1):1-13

Frost HM (1962) Interlamellar thickness in human bone. Clin Orthop Relat Res 24:198-205

Fuller K, Thong JT, Breton BC, Chambers TJ (1994) Automated three-dimensional characterization of the osteoclastic resorption lacunae by stereoscopic scanning electron microscopy. J Bone Miner Res 9(1):17-23

Gebhardt W (1906) Uber funktionelle wichtige Anordungsweisen der feineren und großeren Bauelemente des Wirbeltierknochens, II. Spezieller Teil. Der Bau der Haver'schen Lamellen-systeme und seine funktionelle Bedeutung. Archiv für Entwicklungmechanik der Organismen 20:187-322

Giraud-Guille MM (1988) Twisted plywood architecture of collagen fibrils in human compact bone osteons. Calcif Tissue Int 42(3): $167-180$

Kragstrub J, Nelsen F, Mosekilde L (1983) Thickness of lamellae in normal human iliac trabecular bone. Metab Bone Dis Relat Res 4:291-295

Manelli A, Sangiorgi S, Binaghi E, Raspanti M (2007) 3D analysis of SEM images of corrosion casting using adaptive stereo matching. Microsc Res Tech 70(4):350-354

Marotti G (1973) Decrement in volume of osteoblasts during osteon formation and its effect on the size of the corresponding osteocytes. In: Meunier PJ (ed) Bone histomorphometry. Armour Montagu, Levallois, 1976, pp 385-397 
Marotti G (1993) A new theory of bone lamellation. Calcif Tissue Int 53(S1):47-55

Marotti G, Muglia MA (1988) A scanning electron microscope study of human bony lamellae. Proposal for a new model of collagen lamellar organization. Arch Ital Anat Embriol 93(3):163-175

Marotti G, Ferretti M, Muglia MA, Palumbo C, Palazzini S (1992) A quantitative evaluation of osteoblast-osteocyte relationships on growing endosteal surface of rabbit tibiae. Bone 13(5):363-368

Palumbo C (1986) A three-dimensional ultrastructural study of osteoid-osteocytes in the tibia of chick embryos. Cell Tissue Res 246(1):125-131

Palumbo C, Palazzini S, Zaffe D, Marotti G (1990) Osteocyte differentiation in the tibia of newborn rabbits: an ultrastructural study of the formation of cytoplasmic processes. Acta Anat (Basel) 137(4):350-358

Pazzaglia UE, Andrini L, Di Nucci A (1997) The reaction to nailing or cementing of the femur in rats. A microangiographic and fluorescence study. Int Orthop 21(4):267-273

Pazzaglia UE, Congiu T, Raspanti M, Ranchetti F, Quacci D (2009a) Anatomy of the intracortical canal system: scanning electron microscopy study in rabbit femur. Clin Orthop Relat Res 467:2449-2456

Pazzaglia UE, Congiu T, Ranchetti F, Salari M, Dell'Orbo C (2009b) Scanning electron microscopy study of bone intracortical vessels using an injection and fractured surfaces technique. Anat Sci Int 85:31-27. doi:10.1007/s12565-009-0049-7

Pazzaglia UE, Congiu T, Marchese M, Dell'Orbo C (2010) The shape modulation of osteoblast-osteocyte transformation and its correlation with fibrillar organization in secondary osteons. A SEM study employing the graded osmic maceration technique. Cell Tissue Res 340:533-540

Raspanti M, Guizzardi S, De Pasquale V, Martini D, Ruggeri A (1994) Ultrastructure of heat-deproteinated compact bone. Biomaterials 15(6):433-437
Raspanti M, Guizzardi S, Strocchi R, Ruggeri A (1996) Collagen fibril patterns in compact bone: preliminary ultrastructural observations. Acta Anat (Basel) 155(4):249-256

Reid SA (1986) A study of lamellar organisation in juvenile and adult human bone. Anat Embryol (Berlin) 174(3):329-338

Riggs CM, Lanyon LE, Boyde A (1993a) Functional associations between collagen fibres orientation and locomotor strain direction in cortical bone of the equine radius. Anat Embryol (Berlin) 187(3):231-238

Riggs CM, Vaughan LC, Evans GP, Lanyon LE, Boyde A (1993b) Mechanical implications of collagen fibre orientation in cortical bone of the equine radius. Anat Embryol (Berlin) 187(3): 239-248

Rouiller C, Huber L, Kellenberger E, Rutishauser E (1952) The lamellar structure of the osteon. Acta Anat (Basel) 14(1-2):9-22

Ruth EB (1953) Bone studies. II. An experimental study of the Haversian-type vascular channels. Am J Anat 93(3):429-455

Smith JW (1960) The arrangement of collagen fibres in human secondary osteones. J Bone Joint Surg Br 42-B:588-605

Smith JW (1963) Age changes in the organic fraction of bone. J Bone Joint Surg Br 45:761-769

Wagermaier W, Gupta HS, Gourrier A, Burghammer M, Roschger P, Fratzi P (2006) Spiral twisting of fiber orientation inside bone lamellae. Biointerphases 1(1): 1

Weidenreich F (1923) Knochenstudien 1. Über Aufbau und Entwicklung des Knochens und der Chowauter des Knochengewebes. Z Anat Entwickl Gesch 69:382-466

Weiner S, Traub W, Wagner HD (1999) Lamellar bone: structurefunction relations. J Struct Biol 126:241-255

Ziegler O (1906) Studien über die feinere Struktur des Rőhrenknochens und dessen Polarisation. Dtsch Z Chir 85:248-263 